Historic, Archive Document

Do not assume content reflects current scientific knowledge, policies, or practices. 



\section{THIRD ANNUAL IRIS BARGAINS}

The success of the last two years and the satisfaction of my customers prompts me to make this third offering. Although the list is not as large as last year, it contains exceptional and good values. I will send the same strong divisions as in the past and good measure.

The prices are for the number of roots listed and I can only split the lot if at least 25 of a variety are taken. In such case, the price is in proportion; that is, if 50 of one variety are listed, I will sell 25 at one-half of the price quoted. All quotations are strictly subject to previous sale.

Prices are by express collect.

\begin{tabular}{|c|c|}
\hline & \\
\hline & 5.00 \\
\hline & 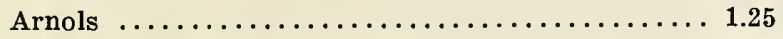 \\
\hline & Argonaut $\ldots \ldots \ldots \ldots \ldots \ldots \ldots \ldots \ldots \ldots \ldots \ldots \ldots \ldots$ \\
\hline & 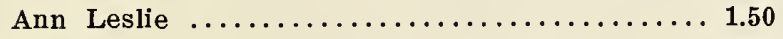 \\
\hline & $\ldots \ldots \ldots \ldots \ldots \ldots \ldots \ldots \ldots \ldots \ldots$ \\
\hline & . \\
\hline & Benbow $\ldots \ldots \ldots \ldots \ldots \ldots \ldots$ \\
\hline & Belladonna (Koehler) .......... \\
\hline & 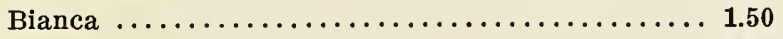 \\
\hline & Black Knight $\ldots \ldots \ldots \ldots \ldots \ldots \ldots \ldots \ldots \ldots$ \\
\hline & Blue Jay $\ldots \ldots \ldots \ldots \ldots \ldots \ldots \ldots \ldots \ldots$ \\
\hline & Blue Lagoon $\ldots \ldots \ldots \ldots \ldots \ldots \ldots \ldots \ldots \ldots$ \\
\hline & Carmencita $\ldots \ldots \ldots \ldots \ldots \ldots \ldots \ldots \ldots \ldots$ \\
\hline & Camelot $\ldots \ldots \ldots \ldots \ldots \ldots \ldots \ldots \ldots \ldots \ldots$ \\
\hline & Chester Hunt $\ldots \ldots \ldots \ldots \ldots \ldots \ldots \ldots \ldots \ldots \ldots$ \\
\hline & 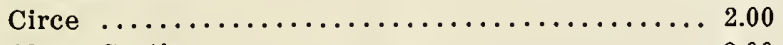 \\
\hline & 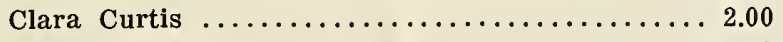 \\
\hline & Clarence Wedge $\ldots \ldots \ldots \ldots \ldots \ldots \ldots \ldots \ldots \ldots \ldots . \ldots \ldots$ \\
\hline & 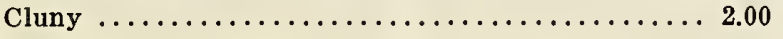 \\
\hline & 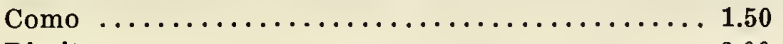 \\
\hline & Dimity $\ldots \ldots \ldots \ldots \ldots \ldots \ldots \ldots \ldots \ldots \ldots \ldots$ \\
\hline & Donna Nook $\ldots \ldots \ldots \ldots \ldots \ldots \ldots \ldots \ldots$ \\
\hline & 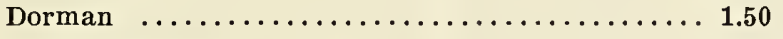 \\
\hline & Dora Longdon $\ldots \ldots \ldots \ldots \ldots \ldots \ldots \ldots \ldots \ldots \ldots \ldots \ldots$ \\
\hline & 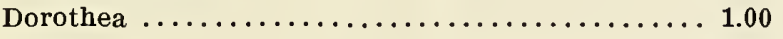 \\
\hline & 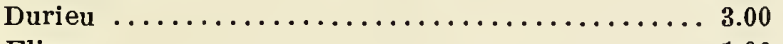 \\
\hline & 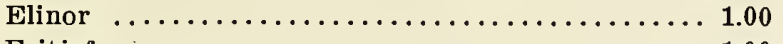 \\
\hline & 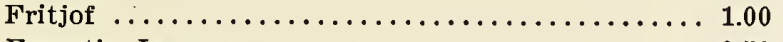 \\
\hline & 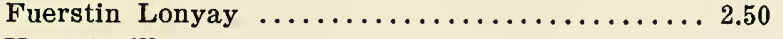 \\
\hline & Hautefeuille $\ldots \ldots \ldots \ldots \ldots \ldots \ldots \ldots \ldots \ldots \ldots \ldots \ldots \ldots \ldots \ldots, 1.2$ \\
\hline & 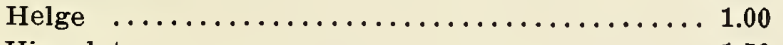 \\
\hline & 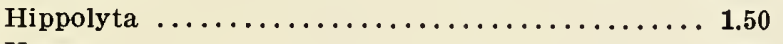 \\
\hline & Hope $\ldots \ldots \ldots \ldots \ldots \ldots \ldots \ldots \ldots \ldots \ldots \ldots \ldots, 1.00$ \\
\hline & 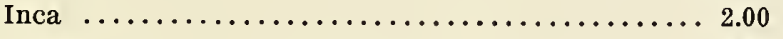 \\
\hline & 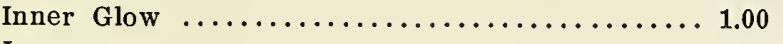 \\
\hline & 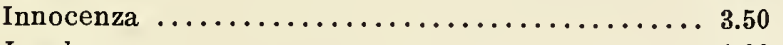 \\
\hline & 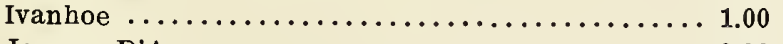 \\
\hline & 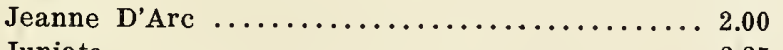 \\
\hline & Juniata $\ldots \ldots \ldots \ldots \ldots \ldots \ldots \ldots \ldots \ldots \ldots \ldots \ldots \ldots \ldots \ldots \ldots \ldots \ldots \ldots \ldots, 2$ \\
\hline & 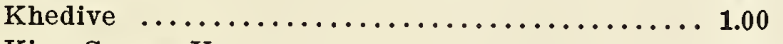 \\
\hline & 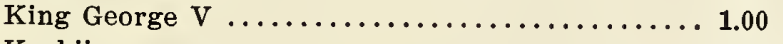 \\
\hline & 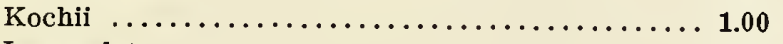 \\
\hline & 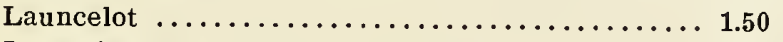 \\
\hline & 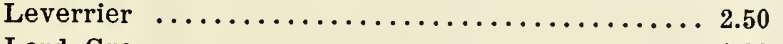 \\
\hline & 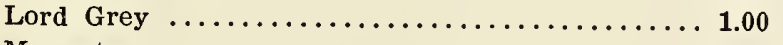 \\
\hline & 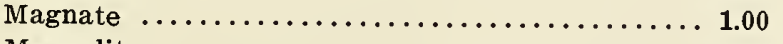 \\
\hline & 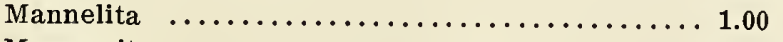 \\
\hline & 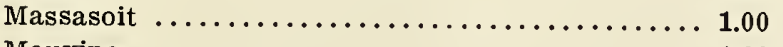 \\
\hline & 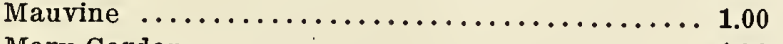 \\
\hline & 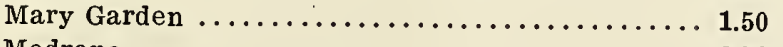 \\
\hline & 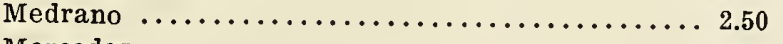 \\
\hline & 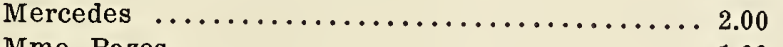 \\
\hline & tes $\ldots \ldots \ldots \ldots \ldots \ldots \ldots \ldots \ldots \ldots \ldots \ldots \ldots$ \\
\hline & $\begin{array}{l}\text { Mme. Boullet } \ldots \ldots \ldots \ldots \ldots \ldots \ldots \ldots \ldots \ldots \ldots \ldots \ldots \ldots \ldots \ldots \ldots \ldots \ldots \ldots \\
\text { Mme. Blanche Pion } \ldots \ldots \ldots \ldots \ldots \ldots \ldots \ldots \ldots \ldots\end{array}$ \\
\hline
\end{tabular}

\begin{tabular}{|c|c|}
\hline & \\
\hline & veigne $\ldots \ldots \ldots \ldots \ldots \ldots \ldots \ldots$ \\
\hline & 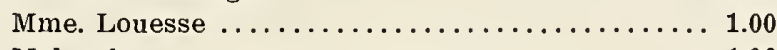 \\
\hline & Iohawk .... \\
\hline & \\
\hline & \\
\hline & ( \\
\hline & \\
\hline & $\ldots \ldots \ldots \ldots \ldots \ldots \ldots \ldots \ldots \ldots \ldots$ \\
\hline & \\
\hline & $\ldots \ldots \ldots \ldots \ldots \ldots \ldots \ldots \ldots \ldots$ \\
\hline & Orage...$\ldots \ldots \ldots \ldots \ldots \ldots \ldots \ldots$ \\
\hline & er Perthuis $\ldots \ldots \ldots \ldots \ldots \ldots \ldots \ldots \ldots$ \\
\hline & 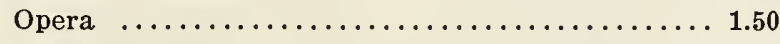 \\
\hline & \\
\hline & s Favorite $\ldots \ldots \ldots \ldots \ldots \ldots \ldots \ldots \ldots$ \\
\hline & 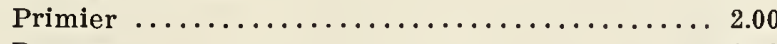 \\
\hline & $\ldots \ldots \ldots \ldots \ldots \ldots \ldots \ldots \ldots \ldots \ldots$ \\
\hline & 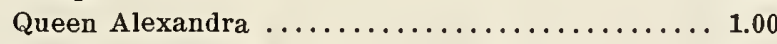 \\
\hline & ay $\ldots \ldots \ldots \ldots \ldots \ldots \ldots \ldots \ldots \ldots \ldots \ldots \ldots \ldots$ \\
\hline & $\ldots \ldots \ldots \ldots \ldots \ldots \ldots \ldots \ldots \ldots \ldots \ldots \ldots$ \\
\hline & 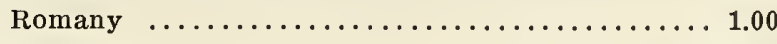 \\
\hline & 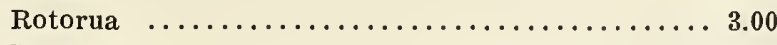 \\
\hline & 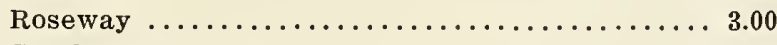 \\
\hline & 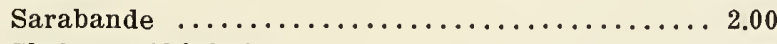 \\
\hline & tain $\ldots \ldots \ldots \ldots \ldots \ldots \ldots \ldots . . \ldots \ldots$ \\
\hline & it $\ldots \ldots \ldots \ldots \ldots \ldots \ldots \ldots \ldots \ldots \ldots \ldots \ldots \ldots$ \\
\hline ( & 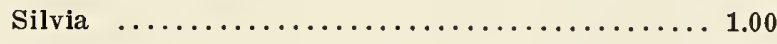 \\
\hline & 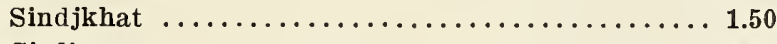 \\
\hline & 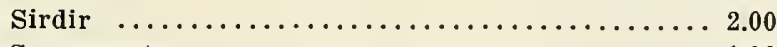 \\
\hline & 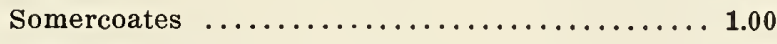 \\
\hline 6 & 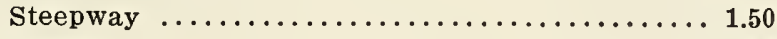 \\
\hline & 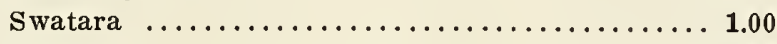 \\
\hline 6 & 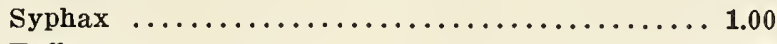 \\
\hline & 1.00 \\
\hline & 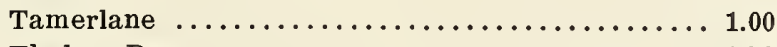 \\
\hline & 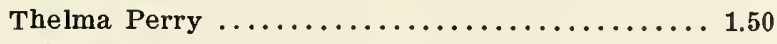 \\
\hline & 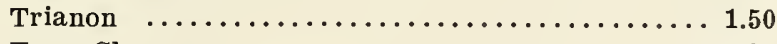 \\
\hline & harm $\ldots \ldots \ldots \ldots \ldots \ldots \ldots \ldots \ldots \ldots \ldots$ \\
\hline & 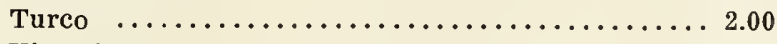 \\
\hline & 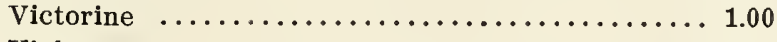 \\
\hline & 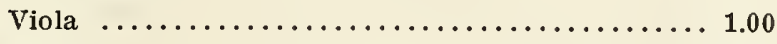 \\
\hline & 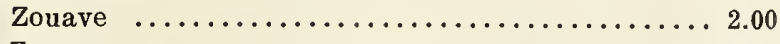 \\
\hline & 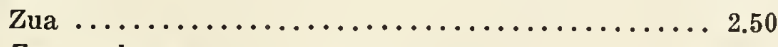 \\
\hline & 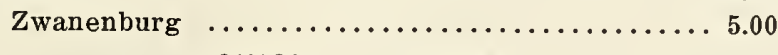 \\
\hline & SINGLE RHIZOMES \\
\hline 4 & e, each..................... \\
\hline 8 & 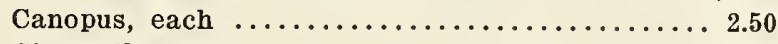 \\
\hline 4 & 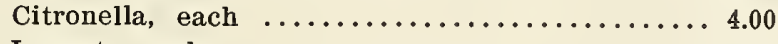 \\
\hline & 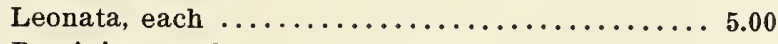 \\
\hline & $\operatorname{ach} \ldots \ldots \ldots \ldots \ldots \ldots \ldots \ldots \ldots \ldots \ldots .6 .00 \ldots$ \\
\hline 10 & 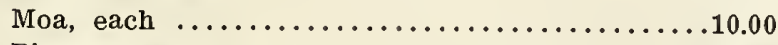 \\
\hline 2 & 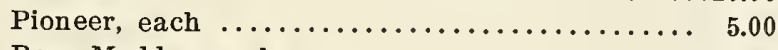 \\
\hline & h $\ldots \ldots \ldots \ldots \ldots \ldots \ldots \ldots \ldots \ldots$ \\
\hline & . \\
\hline & \\
\hline
\end{tabular}


